\title{
NEW OPTICAL AND MERLIN IMAGES OF THE QUADRUPLE GRAVITATIONAL LENS B1422+231
}

\author{
C. E. AKUJOR AND A. R. PATNAIK \\ Max-Planck-Institut für Radioastronomie, Bonn, Germany
}

AND

J. V. SMOKER AND S. T. GARRINGTON

Nuffield Radio Astronomy Labs, Jodrell Bank, U.K.

B1422+231, a multiple component quasar at a redshift of 3.62 , is believed to be a gravitationally lensed system (Patnaik et al. 1992). It has 4 components with maximum image separation of 1.3 arcsec. The three brighter components, $\mathrm{A}, \mathrm{B}$ and $\mathrm{C}$ have similar polarization properties at $8.4 \mathrm{GHz}$. The radio spectra between 5 and $8.4 \mathrm{GHz}$ of $\mathrm{A}, \mathrm{B}$ and $\mathrm{C}$ are similar; since $\mathrm{D}$ is very weak its spectrum is not accurately determined.

We observed $\mathrm{B} 1422+231$ with the NOT; the seeing was 0.55 to 0.60 arcsec. The CCD camera has $520 \times 520$ pixels; each pixel is equivalent to 0.2 arcsec. A calibration field, F873-8 (Stobie et al. 1985) was observed in $\mathrm{V}, \mathrm{R}$ and I bands. The data have been flat-fielded and sky-subtracted. B1422+231 was also observed at $1658 \mathrm{MHz}$ with MERLIN (Thomasson 1986) for $12 \mathrm{hrs}$ in 'phase-reference' mode. The results are summarized in Table 1.

All the components including $\mathrm{D}$ are clearly detected at both radio and optical bands. These optical images are similar to those of Yee \& Ellington (1994) who observed in $r$ and $g$. The spectral indices of A, B, C and D are $-0.42,-0.43,-0.41$ and -0.15 respectively and confirm the turnover in the radio spectrum (Patnaik et al. 1992). No polarization is detected at 1658 $\mathrm{MHz}$.

Patnaik et al. (1992) presented strong evidence for gravitational lensing but this was not as strong for $\mathrm{D}$. Although the radio spectral index of $\mathrm{D}$ is slightly different at radio frequencies, the brightness ratio between the well-separated components $\mathrm{C}$ and $\mathrm{D}$ is similar at both radio and optical frequencies. This is one of the strongest arguments in favor of the lensing hypothesis for all the four components. These are amongst the earliest 

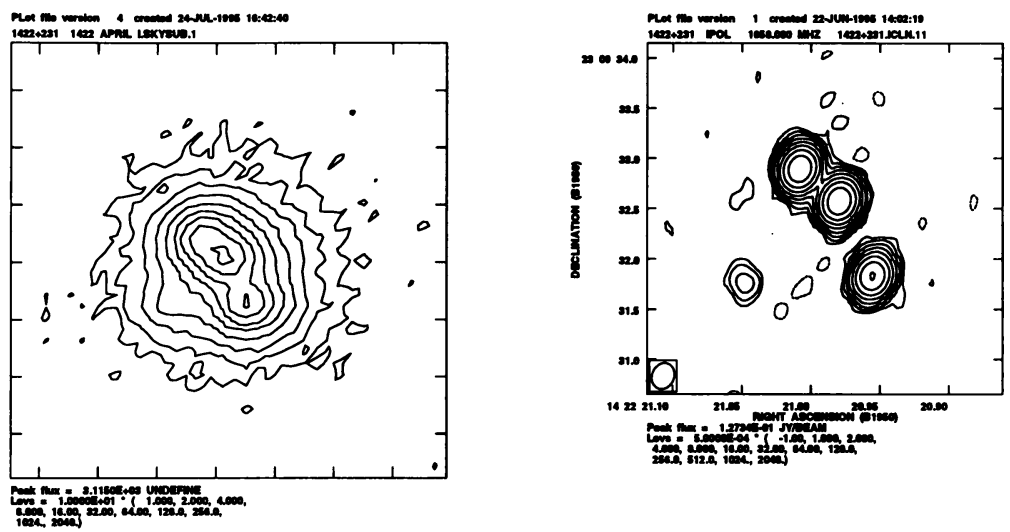

Figure 1. Images of B1422+231: I-band (1993, left); 1.7GHz MERLIN (right).

TABLE 1. The magnitudes of the whole source and magnitude and flux density ratios. The absolute magnitude calibration is not available for B-band; the typical error is $\sim 10 \%$

\begin{tabular}{rcclllll} 
Filter & Obs date & Scan & mag & A & B & C & D \\
\hline B & 93 april23 & $300 \mathrm{~s}$ & - & 1.0 & 1.49 & 0.61 & $\leq 0.025$ \\
V & 92 june30 & $300 \mathrm{~s}$ & $15.42 \pm 0.03$ & 1.0 & 1.35 & 0.60 & 0.028 \\
R & 92 june30 & $300 \mathrm{~s}$ & $15.59 \pm 0.06$ & 1.0 & 1.52 & 0.73 & 0.037 \\
I & 92 june30 & $300 \mathrm{~s}$ & $15.0 \pm 0.1$ & 1.0 & 1.34 & 0.66 & 0.038 \\
I & 93 april23 & $300 \mathrm{~s}$ & $15.2 \pm 0.1$ & 1.0 & 1.20 & 0.52 & 0.027 \\
$1658 \mathrm{MHz}$ & 93 apr09 & $12 \mathrm{hrs}$ & $350.4 \pm 5 \mathrm{mJy}$ & 1.0 & 1.01 & 0.54 & 0.03 \\
\hline
\end{tabular}

observations of this lens system. So, they hopefully provide a standard for further monitoring of the lens images for possible variability.

\section{Acknowledgements}

We are grateful to Prof. A. Ardeberg, Dr. Jensen and NOT staff for support during the observations. CEA acknowledges Swedish NFR, and Av. Humboldt fellowships.

\section{References}

Patnaik, A.R. et al., 1992, MNRAS, 259, 1p

Stobie, R.S., Sagar, R. \& Gilmore, G., 1985, AAS, 60, 503

Thomasson, P., 1986, QJRAS, 27, 413

Yee, H.K.C. \& Ellingson, E., 1984, AJ, 107, 28 